\title{
Las tecnologías de la información y la comunicación aplicadas entre los profesionales de la medicina que realizan tratamiento del dolor
}

\author{
J. Muriel Fernández¹, M. B. García-Cenador,2, N. López-Valverde ${ }^{1}$, C. Muriel² y M. J. Sánchez-Ledesma ${ }^{1,2}$ \\ ${ }^{1}$ Departamento de Cirugía. Facultad de Medicina. Universidad de Salamanca. ${ }^{2}$ Cátedra Extraordinaria del \\ Dolor "Fundación Grünenthal".
}

\section{ABSTRACT}

Target: The revolution in information and communication technologies is the basis on which the advance of health technological innovations and biomedical research has been developed. These advances have been decisive in the improvement of health, since they have involved new forms of medical care that contribute to the increase in life expectancy, pain reduction and disease management. The aim is to assess the level of acceptance of new technologies in physicians who treat pain through specific units.

Material and method: A study was carried out on the use of new information technologies in physicians who perform pain treatment in specific centers. The data was obtained from the elaboration of an online questionnaire that has been sent via email to the students of the Master in Pain Treatment of the USAL. After the systematic collection of information, we performed the statistical analyzes addressing the frequency of the variables and the statistically significant relationships relevant to our study. Later, we carried out a factor analysis, cluster analysis and the construction of new compound variables to synthesize the data. After this identification, the analysis of statistically significant relationships has allowed us to characterize these individuals. Finally, we performed an analysis using a logistic regression methodology through the Binomial Logit model.

The tool used to carry out the online survey was Google Sheets, a tool included within Google Docs, which is designed to work through the Internet and the Web.

Results: The $57 \%$ of the medical professionals who answered our survey are men, while $43 \%$ of the answers belong to women, of Pain Unit [39.46\%),

Muriel Fernández J, García-Cenador MB, López-Valverde N, Muriel C, Sánchez-Ledesma MJ. Las tecnologías de la información y la comunicación aplicadas entre los profesionales de la medicina que realizan tratamiento del dolor. Rev Soc Esp Dolor 2019;26(3):175-183.

\section{RESUMEN}

Objetivos: La revolución de las tecnologías de la información y la comunicación es la base sobre la que se ha desarrollado el avance de las innovaciones tecnológicas sanitarias y la investigación biomédica. Estos avances han sido determinantes en la mejora de la salud, ya que han supuesto nuevas formas de cuidados médicos que contribuyen al aumento de la esperanza de vida, la reducción del dolor y la gestión de las enfermedades. Se pretende valorar el nivel de aceptación de las nuevas tecnologías en médicos que traten el dolor a través de unidades específicas.

Material y métodos: Se llevó a cabo un estudio sobre el uso de las nuevas tecnologías de la información en médicos que realizan tratamiento del dolor en centros específicos. Los datos se obtuvieron a partir de la elaboración de un cuestionario online que ha sido enviado a través del correo electrónico a los alumnos del Máster en Tratamiento del Dolor de la USAL. Tras la recogida sistemática de la información, hemos realizado los análisis estadísticos abordando la frecuencia de las variables y las relaciones estadísticamente significativas relevantes para nuestro estudio. Posteriormente realizamos un análisis de factores, análisis de clúster y la construcción de nuevas variables compuestas para sintetizar los datos. Tras esta identificación, el análisis de las relaciones estadísticamente significativas nos ha permitido caracterizar a estos individuos. Finalmente, hemos realizado un análisis utilizado una metodología de regresión logística a través del modelo Logit binomial.

La herramienta utilizada para la realización de la encuesta online fue Google Sheets, herramienta englobada dentro de Google Docs, que está diseñada para trabajar a través de internet y de la web.

Resultados: El $57 \%$ de los profesionales médicos que contestaron a nuestra encuesta son hombres, mientras que el $43 \%$ de las respuestas pertenecen a mujeres, de

Recibido: 26-09-2018

Aceptado: 20-01-2019

Correspondencia: Jorge Muriel Fernández murimuriel@gmail.com 
Palliative Care [21.35\%] and in the Centers of Primary Care (CAP), Adding this data we obtain that $75.94 \%$ of professionals work in public entities, $9.72 \%$ in private entities and $11.05 \%$ in both. $79.5 \%$ of the surveying professionals do not offer their mobile phone to patients. More than $83 \%$ of the professionals surveyed use very frequently (29.5\%) or Frequently [54.2\%) guidelines / medical protocols in their clinical practice; More than $55 \%$ of the professionals surveyed "never use computers or Information and Communication Technologies for the remote realization of diagnostic evaluation or prescription of therapeutic measures". However, $40 \%$ of the respondents declare that they have done telemedicine.

Conclusions: In general, the data show us a beneficial conception of the applications of Information and Communication Technologies for clinical practice in relation to the digitalization and distribution of clinical information. In addition, given the clinical experience of professionals, the need for organizational changes associated with new computer systems is confirmed.

Key words: Information technologies, pain units, clinical practice, protocols, telemedicine.
Unidad del Dolor [39,46\%), Cuidados Paliativos [21,35\%] y en los Centros de Atención Primaria (CAP). Agregando estos datos obtenemos que el 75,94\% de los profesionales trabajan en entidades públicas, el 9,72\% en entidades privadas y el 11,05 \% en ambas. El 79,5\% de los profesionales encuestados no ofrecen su teléfono móvil a los pacientes. Más del $83 \%$ de los profesionales encuestados utilizan muy frecuentemente $[29,5 \%$ ) o frecuentemente $[54,2 \%$ ) directrices/protocolos médicos en su práctica clínica; más del $55 \%$ de los profesionales encuestados "Nunca utilizan ordenadores o tecnologías de la información y la comunicación para la realización remota de evaluación diagnóstica o prescripción de medidas terapéuticas". No obstante, un $40 \%$ de los encuestados declaran que han realizado telemedicina.

Conclusiones: En general, los datos nos muestran una concepción beneficiosa de las aplicaciones de las tecnologías de la información y la comunicación para la práctica clínica en lo relativo a la digitalización y distribución de la información clínica. Además, se constata, dada la experiencia clínica de los profesionales, la necesidad de cambios organizativos asociados a los nuevos sistemas informáticos.

Palabras clave: Tecnologías de la información, unidades de dolor, práctica clínica, protocolos, telemedicina.

\section{INTRODUCCIÓN}

Con la aparición de internet, mediante un computador, un módem y una línea telefónica y hasta con un teléfono móvil, ya es posible acceder a toda la información que uno pueda necesitar, a un costo muy modesto y sin necesidad de las tradicionales bibliotecas de costos astronómicos para poder estar medianamente bien dotadas de libros y revistas científicas.

A inicios de la década de 1990, con la introducción de nuevas facilidades de interconexión y herramientas gráficas simples para el uso de la red, se inició el auge que actualmente le conocemos a internet. Este crecimiento masivo trajo consigo el surgimiento de un nuevo perfil de usuarios, en su mayoría de personas comunes no ligadas a los sectores académicos, científicos y gubernamentales.

La revolución de las tecnologías de la información y la comunicación es la base sobre la que se ha desarrollado el avance de las innovaciones tecnológicas sanitarias y la investigación biomédica. Estos avances han sido determinantes en la mejora de la salud, ya que han supuesto nuevas formas de cuidados médicos que contribuyen al aumento de la esperanza de vida, la reducción del dolor y la gestión de las enfermedades.

La Organización foro Economic Cooperation and Development (1) también destaca la potencialidad de las tecnologías de la información y la comunicación para la mejora en la actuación de los sistemas de salud. Anderson y cols. (2) sugieren que la adopción de estas tecnologías podría facilitar la disminución del gasto y el aumento de la calidad de los servicios.

Centrándonos en la evaluación del dolor, hay que decir que, en los últimos años, han incrementado los instrumentos electrónicos para evaluar la experiencia dolorosa. Varias son las ventajas que este hecho reporta. Una de las más importantes es la posibilidad de registrar las variables en tiempo real, mitigando de esta forma los efectos de memoria en las evaluaciones retrospectivas y, por consiguiente, incrementando la precisión de las respuestas dadas por el paciente.

Como espacio de comunicación, internet podría transformar los procesos de interacción entre los diferentes agentes del sistema sanitario, y entre estos y las organizaciones donde desarrollan su actividad. Todo ello facilitaría el aumento de los flujos de información, mejoraría la accesibilidad al sistema y podría generar comunidades en red de apoyo mutuo [3].

\section{MATERIAL Y MÉTODOS}

La información se obtuvo a partir de la elaboración de un cuestionario online que ha sido enviado a través del correo electrónico a 531 alumnos del Máster en Tratamiento del Dolor.

El número de respuestas obtenidas ha sido del 73,25 \% (389 respuestas), siendo no válidas 19: 12 por contestación de forma incorrecta, 4 por no estar de acuerdo con el cuestionario y 3 por pedir una com- 
pensación económica, siendo los cuestionarios válidos para realizar el estudio 370 , el trabajo de campo se realizó durante los meses de julio a septiembre del 2017.

Para superar las cuestiones relacionadas con la privacidad y la confidencialidad de la encuesta, el lanzamiento de los correos electrónicos fue realizado por la administración del máster, mientras que las respuestas obtenidas eran almacenadas en los servidores de la Cátedra Extraordinaria del Dolor "Fundación Grünenthal" de la Universidad de Salamanca.

Los correos electrónicos constaban de un texto en el que se incluía una explicación del proyecto de investigación, una petición de colaboración y un enlace al cuestionario.

Cada correo electrónico contenía un enlace a la encuesta, lo que garantiza la unicidad de respuesta. La herramienta de encuestas online está diseñada para trabajar a través de la web, de manera que tanto la recogida de datos como su posterior descarga se hace de manera online.

Desde un punto de vista metodológico, en nuestro estudio podemos identificar tres etapas: el diseño de los instrumentos, la recogida sistemática de la información y el análisis estadístico realizado. Así, en función de las preguntas de investigación propuestas, las hipótesis planteadas y los objetos de estudio seleccionados, hemos diseñado dos tipos de instrumentos.

En el caso de los actores objeto de estudio, hemos diseñado un cuestionario que ha sido construido teniendo en cuenta las características del medio a utilizar: internet.

Estos instrumentos metodológicos nos han permitido una recogida sistemática de la información. Por un lado, la matriz de criterios nos ha facilitado la identificación de los ítems seleccionados mediante la observación directa de las webs.

Tras la recogida sistemática de la información, hemos realizado los análisis estadísticos siguiendo el esquema que exponemos a continuación. En primer lugar, abordamos la frecuencia de las variables y las relaciones estadísticamente significativas relevantes para nuestro estudio. En segundo lugar, mediante análisis de factores, análisis de clúster y la construcción de nuevas variables compuestas, hemos sintetizado los datos. Esta reducción de la información nos ha permitido la identificación de los individuos en grupos homogéneos internamente y heterogéneos externamente. Tras esta identificación, el análisis de las relaciones estadísticamente significativas nos ha permitido caracterizar a estos individuos. Finalmente, hemos realizado un análisis causal que nos permite estimar la presencia o ausencia de una característica según los valores de un conjunto de variables predictores o independientes que se asocian estadísticamente. Para realizar este tipo de análisis hemos utilizado una metodología de regresión logística a través del modelo Logit binomial.

Además de estas cuestiones, antes de abordar las metodologías de cada uno de los estudios realizados, presentamos algunas consideraciones generales sobre la aplicación Google Sheets, perteneciente al grupo ofimático de Google Docs, la herramienta de encuestas online que hemos utilizado. Esta herramienta está diseñada para trabajar a través de internet y de la web.
El diseño, la implementación y el envió de los cuestionarios, así como la recepción y almacenamiento de los datos, se realiza totalmente online a través de las aplicaciones y servidores de Google. El proceso de elaboración de los cuestionarios comienza con la elección del tipo de envío que se quiere llevar a cabo; la herramienta dispone de dos opciones: cuestionarios identificados (aquellos que se envían a través del correo electrónico a cada uno de los individuos de la población objeto de estudio] y cuestionarios no identificados [aquellos cuestionarios que son colocados en diferentes sitios web a los que los individuos de la población objeto de estudio pueden acceder]. Una vez seleccionado el tipo de encuesta que se quiere realizar, comienza la fase de diseño y de implementación; para ello, la herramienta de encuestas online dispone de una amplia batería de formatos y opciones que facilitan la construcción del cuestionario. Una vez realizado este paso, el cuestionario está listo para ser enviado a las direcciones de correo electrónico disponibles, en el caso de que la encuesta sea identificada.

En el caso de los cuestionarios identificados, para superar las cuestiones relacionadas con la privacidad y confidencialidad de los correos electrónicos y de los datos recibidos, hemos separado el proceso de envío de los cuestionarios del proceso de recogida y almacenamiento de los datos. De esta manera, cada correo electrónico era identificado mediante una clave que permitía garantizar la unicidad de la respuesta, manteniendo el anonimato de la persona que contestaba. Además, el fichero que contiene los datos de los correos electrónicos permanece siempre en poder de su propietario, sin que sea necesario la cesión del mismo.

\section{RESULTADOS}

\section{Características demográficas y sociales}

La comparación de los datos agregados nos facilita los siguientes resultados en función del género: observamos que existe un diferencial entre hombres (244) y mujeres (126) de las respuestas válidas (Tabla I].

Los resultados nos muestran que esta diferencia entre ambos géneros tenderá a modificarse debido a la incorporación masiva de la mujer a la profesión. En este sentido, los porcentajes de hombres son siempre superiores respecto al de mujeres, excepto en menores de 30 años. Por último, se constata que menos de un tercio de los profesionales son mayores de 50 años.

En la Tabla II, que incluye a todos los individuos que han contestado a la encuesta, podemos observar dónde los profesionales médicos desarrollan su actividad según su titularidad. La distribución de los porcentajes y las combinaciones reflejan la diversidad en los servicios de los distintos sistemas de salud. No obstante, podemos destacar la importancia de los centros de titularidad y gestión pública, unidad del dolor [39,46 \%), cuidados paliativos $(21,35 \%$ y en los centros de atención primaria (CAP). Agregando estos datos obtenemos que el $75,94 \%$ de los profesionales trabajan en entidades públicas, el 9,72 \% en entidades privadas y el $11,05 \%$ en ambas. 
TABLA I

CARACTERÍSTICAS DEMOGRÁFICAS (SEXO Y EDAD) DE LOS PROFESIONALES MÉDICOS QUE CONTESTARON A LA ENCUESTA (NÚMERO/ PORCENTAJES \%]

\begin{tabular}{|c|c|c|}
\hline & Hombre [57\%] & Mujer $[43 \%]$ \\
\hline $\begin{array}{c}\text { Menor de } 30 \\
\text { años }\end{array}$ & $6(2,86)$ & $12(7,50)$ \\
\hline $\begin{array}{c}\text { Entre } 31 \text { y } 40 \\
\text { años }\end{array}$ & $108(51,42)$ & $82[51,25)$ \\
\hline $\begin{array}{c}\text { Entre 41 y } 50 \\
\text { años }\end{array}$ & $78(37,15)$ & $54(33,75)$ \\
\hline $\begin{array}{c}\text { Mayor de } 51 \\
\text { años }\end{array}$ & $18(8,57)$ & $12[7,50)$ \\
\hline
\end{tabular}

TABLA II

DÓNDE REALIZAN LA ACTIVIDAD EL PROFESIONAL (PORCENTAJES \%)

\begin{tabular}{|c|c|}
\hline Unidad de Dolor (UD) & 39,46 \\
\hline Cuidados paliativos & 21,35 \\
\hline CAP o similar & 15,13 \\
\hline Públicos (no UD) & 3,25 \\
\hline Privado & 9,72 \\
\hline Privado y UD & 11,05 \\
\hline
\end{tabular}

Si abordamos solo aquellos profesionales que han declarado que desarrollan su actividad en un único lugar, observamos que el 7,8 \% lo hacen en un Hospital, el 15,1 \% en un Centro de Atención Primaria.

Existe un comportamiento diferencial que nos muestra que son los profesionales con mayores edades los que en un porcentaje mayor desarrollan su actividad profesional en diferentes lugares de trabajo. Mientras que los profesionales más jóvenes desarrollan en un porcentaje mayor su actividad en un único lugar de trabajo.

Otra cuestión importante que caracteriza a los profesionales médicos es su especialidad, es decir, el área de conocimiento de la medicina que abordan en su práctica profesional. El nacimiento de nuevas especialidades (dolor y cuidados paliativos) está asociado con el desarrollo de la medicina como ciencia y la necesidad de acotar y profundizar en aspectos determinados de la salud en el paciente crónico. El 68,1\% de los médicos encuestados pertenecen a especialidades médicas, es decir, aquellas que no realizan intervenciones quirúrgicas. Dentro de estas, destacan la especialidad de Anestesiología [34,5 \%), Medicina Familiar y Comunitaria a [7,8\%) y Medicina interna [6,6\%). Un 22,1\% de los profesionales señalan que pertenecen a especialidades que abordan cuestiones transversales a las especialidades quirúrgicas y médicas.

Por último, además de la especialidad, hemos abordado las tareas que realizan los profesionales médicos en su actividad profesional. El 53,6 \% de los encuestados realizan únicamente tareas relacionadas con la práctica asistencial en el tratamiento del dolor, porcentaje que aumenta hasta el $90,2 \%$ en el caso de combinar la actividad asistencial con otras tareas (por ejemplo, realizar anestesia y tratamiento del dolor). Tan solo un 4,9\% de los profesionales médicos se dedican exclusivamente a la Planificación/Dirección/Gestión en las Unidades del Dolor. Este porcentaje disminuye a menos de un $3 \%$ en los casos en los que se dedican con exclusividad a la investigación o a la docencia.

\section{Usos del teléfono móvil relacionados con la salud}

El 79,5\% de los profesionales encuestados no ofrecen su teléfono móvil a sus pacientes, lo que nos indica que su uso en la práctica profesional es escaso. Además, las prácticas de los profesionales que sí ofrecen su teléfono móvil están relacionadas con la comunicación oral e instantánea, ya que el 76,1\% de los profesionales atienden por norma las llamadas de los pacientes. Tan solo un 11,6 \% de los profesionales adoptan prácticas diferentes a la conversación inmediata respondiendo en función del mensaje grabado $[11,6 \%$ ) o mediante SMS (1\%).

Los profesionales médicos de más de 41 años utilizan el teléfono móvil para comunicarse con sus pacientes más frecuentemente que el resto de profesionales. Además, son los profesionales de anestesia y cuidados paliativos, cuya especialidad no está relacionada con la medicina familiar y comunitaria, los que más utilizan esta tecnología. Por tanto, la explicación a esta tendencia la hemos de encontrar en el contexto del tipo de trabajo donde realizan sus actividades.

Existe una relación significativa entre el número de lugares donde trabaja el profesional y el uso del móvil para comunicarse con los pacientes. Los profesionales que trabajan en más de un lugar tienden a utilizar el móvil más que aquellos que realizan su actividad profesional en un único lugar.

Si segmentamos nuestras respuestas en aquellos profesionales que tan solo trabajan en una institución existe una relación significativa entre el tipo de organización y el uso del móvil. De nuevo son los profesionales médicos que desarrollan su actividad en clínicas y consultorios los que utilizan el teléfono móvil para comunicarse con sus pacientes; existiendo una tendencia estadística en la que destaca la titularidad privada.

\section{Protocolos y modo de información en la práctica clínica}

El uso de protocolos médicos de dolor en la práctica clínica es un buen ejemplo de tratamiento de la información en el ámbito sanitario. Los protocolos describen el proceso en la atención de una enfermedad para mejorar la rapidez en el diagnóstico, llevar a cabo el tratamiento, y hacer menos costoso el proceso de atención, tanto para el paciente como para la entidad prestadora de salud. 
El acceso a estas directrices supone una gestión de la información que facilita a los médicos su práctica clínica.

Más del $83 \%$ de los profesionales encuestados utilizan muy frecuentemente [29,5\%] o frecuentemente [54,2 \%) directrices/protocolos médicos en su práctica clínica; mientras que el 12,8 \% declaran que raramente los utilizan y tan solo el 2,1 \% señalan que nunca los han utilizado.

Los profesionales más jóvenes utilizan con mayor frecuencia las directrices/protocolos médicos en la práctica clínica en una proporción mayor que aquellos profesionales con más edad. Sin embargo, no existen diferencias significativas entre la especialidad de los profesionales, ni entre las entidades proveedoras de servicios y su titularidad con el uso de protocolos médicos.

Existe una relación significativa entre el uso de internet y el uso de protocolos médicos. Aquellos profesionales que usan internet más frecuentemente también utilizan protocolos médicos con más frecuencia. Podemos interpretar que el uso de internet como una nueva fuente de información para el profesional médico viene acompañado con la necesidad de articular el conocimiento disponible en guías que faciliten su aplicación. Por tanto, un mayor uso de internet hará necesaria la existencia de nuevas formas de abordar la información disponible para que sea manejable. Por último, la disponibilidad de que este tipo de protocolos esté online es considerada muy útil por el 62,3\% de los encuestados y útil por el 32,8 \%. Tan solo un 2,3 \% de los profesionales consideran poco útil está medida.

Las tecnologías de la información y la comunicación aplicadas a los sistemas de información en la práctica clínica son una de las principales innovaciones dentro del sistema sanitario. La Tabla III muestra algunas de estas aplicaciones.

Con excepción del envío de SMS para recordar la toma de medicación en supuestos muy concretos, más del $50 \%$ de los profesionales encuestados han utilizado o utilizarían las tecnologías de la información y la comunicación en su práctica clínica para desarrollar acciones relacionadas con la gestión de la información. En el caso de los procesos relacionados con la consulta de registros clínicos dentro de la propia institución y consulta de bases de datos y sistemas de información, el porcentaje del 9,7\%, llamando la atención que un porcentaje superior al $50 \%$ si fuera posible las utilizaría.

Estos resultados contrastan con los porcentajes obtenidos con el envío de recetas o informes con un medio tradicional como el correo postal. El 66,3\% de los profesionales no utilizaría el correo postal para el envío de recetas. Este porcentaje se disminuye hasta el $49,6 \%$ en el caso del envío de partes oficiales o informes médicos.

Por tanto, según los datos obtenidos, en el apartado de si fuera posible, los profesionales médicos tienen una buena predisposición para el uso de las tecnologías de la información y la comunicación en los procesos relacionados con la gestión de la documentación médica.

Existe una relación significativa entre la utilización de este tipo de tecnologías para el seguimiento y la consulta de información médica y la edad. En este caso, como en el uso de internet, son los profesionales con menor edad los que responden positivamente ante el uso o posible uso de este tipo de tecnologías.

En el caso de las tecnologías de la información y la comunicación asociadas a la consulta de información médica, al envío de recetas electrónicas y al envío de SMS para recordar tomar la medicación, son los profesionales médicos que trabajan en los centros de atención primaria los que mayor predisposición tienen a trabajar con este tipo de tecnologías.

La estructura y la actividad que se desarrolla en cada uno de estos centros condicionan la implantación y el uso de las tecnologías de la información y la comunicación. Los hospitales (unidades de dolor) son centros donde se presta una atención especializada y puntual a los usuarios con una estructura vertical, donde los profesionales médicos que se encuentran en puestos de dirección poseen un gran poder sobre las decisiones que se toman en su servicio.

En cambio, en los centros de atención primaria se prestan unos servicios médicos que generalmente van asociados a una visión más integral del paciente, por tanto, la gestión e integración de la información clínica

TABLA III

MODO DE INFORMACIÓN EN LA PRÁCTICA CLÍNICA (PORCENTAJES \%)

\begin{tabular}{|c|c|c|c|}
\hline $\begin{array}{c}\text { Seguimiento de consultas por vía electrónica } \\
\text { (internet, SMS) }\end{array}$ & $\begin{array}{c}\text { Sí, la la utilizaría si } \\
\text { fuese posible }\end{array}$ & No la utilizaría \\
\hline $\begin{array}{c}\text { Envío de recetas por vía electrónica (internet, otro } \\
\text { tipo de red) }\end{array}$ & $3,7,7,5$ & 38,8 \\
\hline $\begin{array}{c}\text { Envío de partes oficiales o informes médicos por } \\
\text { vía electrónica }\end{array}$ & 7,0 & 54,2 & 12,1 \\
\hline $\begin{array}{c}\text { Envío de SMS para recordar la toma de } \\
\text { medicación }\end{array}$ & 03,4 & 49,6 \\
\hline $\begin{array}{c}\text { Consulta de registros clínicos informatizados } \\
\text { dentro de su propia institución }\end{array}$ & 18,4 & 34,8 & 65,2 \\
\hline
\end{tabular}


tiene mucha importancia para los profesionales. Además, el hecho de que su estructura sea más horizontal, menos jerárquica, facilita la implantación de sistemas de información que ayuden a compartir la información disponible sobre el paciente.

\section{Telemedicina}

Las tecnologías de la información y la comunicación, además de facilitar el acceso, la distribución y la generación de información, también pueden ser utilizadas para la provisión de servicios sanitarios a través de la telemedicina (Tabla IV), entendida como la utilización de ordenadores o tecnologías de la información y la comunicación para la realización remota de la actividad médica.

Más del 55 \% de los profesionales encuestados nunca utilizan ordenadores o tecnologías de la información y la comunicación para la realización remota de evaluación diagnóstica o prescripción de medidas terapéuticas. No obstante, un $40 \%$ de los encuestados declaran que han realizado telemedicina (muy frecuentemente/frecuentemente/raramente).

Estos datos reflejan la escasa implantación de la telemedicina. A pesar de esta escasa implantación, de aquellos que han utilizado la telemedicina un 19,3\% lo ha considerado muy útil, un $57,2 \%$ útil y un $8,4 \%$ poco útil.
Existe una relación significativa entre la especialidad del profesional y la práctica de la telemedicina: hay un porcentaje más elevado de profesionales de cuidados paliativos entre los que sí utilizan la telemedicina. También existe una relación significativa con el tipo de centro donde trabajan, entre los que utilizan la telemedicina los profesionales que trabajan en hospitales son los más numerosos.

Existe una relación significativa entre la edad de los profesionales médicos y el uso de la telemedicina. En este caso, son los profesionales con mayor edad los que la realizan. La explicación la podemos encontrar en el deseo de innovación, en las estructuras jerárquicas de las organizaciones, especialmente en los hospitales.

El uso de la telemedicina cada vez viene más acompañado del uso de otras tecnologías de la información y la comunicación que permiten una interacción más intensa y continuada con los pacientes, que va más allá de la evaluación diagnóstica y la prescripción de medidas terapéuticas. En este sentido, existe una relación significativa y positiva entre la telemedicina y el número de actividades que realiza el médico por internet lya sea de contacto o búsqueda de información).

Los datos recogidos a través del grado de acuerdo de los profesionales con afirmaciones relativas a las tecnologías de la información y la comunicación nos ofrecen una impresión favorable a su uso, dado los resultados en la Tabla $\mathrm{V}$.

TABLA IV

UTILIZA ORDENADORES O TECNOLOGÍAS DE LA INFORMACIÓN Y LA COMUNICACIÓN PARA LA REALIZACIÓN REMOTA (TELEMEDICINA) (PORCENTAJES \%)

\begin{tabular}{|c|c|c|c|c|}
\hline & $\begin{array}{c}\text { Muy } \\
\text { Frecuentemente }\end{array}$ & Frecuentemente & Raramente & Nunca \\
\hline Evaluación diagnóstica & 5,6 & 9,4 & 28,8 & 56,2 \\
\hline $\begin{array}{c}\text { Prescripción de medidas } \\
\text { terapéuticas }\end{array}$ & 4,4 & 9,0 & 27,9 & 58,7 \\
\hline Otras & 3,8 & 11,9 & 11,8 & 72,5 \\
\hline
\end{tabular}

TABLA V

GRADO DE ACUERDO CON LAS SIGUIENTES INFORMACIONES (PORCENTAJES \%)

\begin{tabular}{|c|c|c|}
\hline $\begin{array}{c}\text { En } \\
\text { clínico del paciente es muy útil para la práctica clínica }\end{array}$ & De acuerdo & desacuerdo \\
\hline $\begin{array}{c}\text { Soy favorable a la creación de un registro informatizado único por } \\
\text { paciente, que sea accesible a través de internet al profesional de la salud, } \\
\text { independientemente del centro donde se atienda al paciente }\end{array}$ & 95,6 & 94,3 \\
\hline $\begin{array}{c}\text { Con el uso masivo de las tecnologías de la información y la comunicación existe } \\
\text { un mayor control de los errores }\end{array}$ & 78,8 & 5,7 \\
\hline $\begin{array}{c}\text { Mi práctica clínica me dice que es tan importante innovar en las cuestiones } \\
\text { organizativas de la unidad del dolor y la institución, como invertir en nuevas } \\
\text { prácticas a través del uso de las tecnologías de la información y comunicación }\end{array}$ & 77,6 & 22,4 \\
\hline
\end{tabular}


Más del $95 \%$ de los profesionales encuestados está totalmente de acuerdo, por un lado, con que la aplicación de las tecnologías de la información y la comunicación a las cuestiones relacionadas con la existencia de la historia clínica es muy útil para la práctica clínica y, por otro lado, que este tipo de aplicaciones han de venir acompañadas de innovaciones organizativas.

El 94,3\% de los profesionales está totalmente de acuerdo con la creación de un registro informatizado único por paciente con dolor crónico, que sea accesible a través de internet al profesional de la salud, independientemente del centro donde se atienda al paciente. Además, el 78,8 \% están parte de acuerdo en que con el uso masivo de las tecnologías de la información y la comunicación existe un mayor control de los errores.

En general, los datos nos muestran una concepción beneficiosa de las aplicaciones de las tecnologías de la información y la comunicación para la práctica clínica en lo relativo a la digitalización y distribución de la información clínica. Además, se constata, dada la experiencia clínica de los profesionales, la necesidad de cambios organizativos asociados a los nuevos sistemas informáticos.

Por último, alrededor del 77,6 \% de los profesionales está en desacuerdo con que el uso de las tecnologías de la información y la comunicación introduzca una mayor burocracia y tenga una débil influencia en la mejoría de la práctica clínica.

Si abordamos la dimensión relacionada con la valoración de las tecnologías de la información y la comunicación que realizan los profesionales encuestados (Tabla VI), se observa que existe un porcentaje mayor de lo esperado de médicos en red que están totalmente de acuerdo con que la existencia de datos informatizados que permita ver la evolución del estado clínico del paciente es muy útil para la práctica clínica, con que el uso masivo de las tecnologías de la información y la comunicación existe un mayor control de los errores, y que es tan importante innovar en las cuestiones organizativas de los servicios y la institución como invertir en nuevas prácticas a través del uso de estas tecnologías.

\section{DISCUSIÓN}

Los médicos que tratan el dolor como enfermedad se enfrentan a los beneficios y los riesgos que supone la introducción de las tecnologías de la información y la comunicación, especialmente internet, en el ámbito de la salud (4-9). El acceso y el uso de estas nuevas tecnologías podría tener consecuencias tanto sobre su práctica médica como sobre la configuración de dichas tecnologías en el contexto de las organizaciones donde desarrollan su trabajo $(10,11)$ y su acceso a unas fuentes de información especializada que les permita una actualización constante de sus conocimientos (12-14).

Internet también puede ser configurado como una herramienta para la asistencia médica. Los profesionales médicos con labor asistencial, docente e investigadora pueden disponer de sistemas de información que les permitan construir y consultar el historial médico del paciente. Esta nueva gestión de la información clínica podría transformar tanto su práctica clínica como su

TABLA VI

VALORACIÓN DE LAS TECNOLOGÍAS DE LA INFORMACIÓN Y DE LA COMUNICACIÓN DEL MÉDICO EN UD (DIMENSIONES, PORCENTAJE DE MÉDICOS Y SIGNIFICATIVIDAD CHI CUADRADO)

\begin{tabular}{|c|c|c|c|c|}
\hline Dimensiones & $\begin{array}{l}\text { Intensidad, experiencia y utilidad en el uso } \\
\text { de internet }\end{array}$ & $\begin{array}{l}\text { Médicos } \\
\text { en UD }\end{array}$ & $\begin{array}{l}\text { Médico } \\
\text { tradicional }\end{array}$ & Significatividad \\
\hline \multicolumn{4}{|c|}{$\begin{array}{c}\text { La existencia de datos informatizados que permitan ver la evolución del estado clínico del } \\
\text { paciente es muy útil para la práctica clínica }\end{array}$} & 0,033 \\
\hline & Totalmente de acuerdo & 69,5 & 63,1 & \\
\hline & En parte de acuerdo & 28,3 & 35,1 & \\
\hline & En desacuerdo & 1,6 & 1,5 & \\
\hline \multicolumn{4}{|c|}{$\begin{array}{c}\text { Con el uso masivo de las tecnologías de la información y la comunicación existe un mayor } \\
\text { control de los errores }\end{array}$} & 0,002 \\
\hline & Totalmente de acuerdo & 22,3 & 16,7 & \\
\hline & En parte de acuerdo & 57,5 & 60,5 & \\
\hline & En desacuerdo & 19,2 & 22,8 & \\
\hline \multicolumn{4}{|c|}{$\begin{array}{c}\text { Mi práctica clínica me dice que es tan importante innovar en las cuestiones organizativas } \\
\text { de los servicios y la institución, como invertir en nuevas prácticas a través del uso de las } \\
\text { tecnologías de la información y comunicación }\end{array}$} & 0,000 \\
\hline & Totalmente de acuerdo & 68,2 & 54,5 & \\
\hline & En parte de acuerdo & 3408 & 43,0 & \\
\hline & En desacuerdo & 1,0 & 2,4 & \\
\hline
\end{tabular}


manera de relacionarse con el paciente y con otros profesionales sanitarios. La escasa formación de los profesionales médicos en el uso de las tecnologías de la información y la comunicación y las barreras asociadas con la falta de confidencialidad y seguridad de los datos puede suponer un problema a la hora de utilizar este tipo de recursos (15). Además, estos sistemas de información también podrían tener repercusiones sobre el control o la autonomía de los profesionales en su práctica diaria, lo que podría tener un impacto en la calidad, en la eficiencia y en los costes de su institución.

Las consecuencias reales en cualquier sector de actividad de la introducción y uso de las tecnologías sobre la cualificación, las actividades, la organización y los resultados del trabajo dependen tanto de las características formativas, de las habilidades de aprendizaje, de la experiencia y de la interacción de las organizaciones con su entorno, como del patrón de competitividad económico y social. En este sentido, durante la introducción de un proceso de innovación tecnológico se establece un complejo tejido de interacciones organizativas, productivas, laborales, institucionales y culturales que condicionan el propio proceso en función de la dirección de estas interrelaciones. Por tanto, el impacto de las tecnologías solo se puede comprender a partir del análisis de estas complejas interacciones dentro del sistema social en el que se producen $(14,16)$. La identificación, caracterización y determinación de los usos intensivos de internet nos muestra que los médicos, encuestados no son ajenos a esta realidad.

El uso de las tecnologías de la información y la comunicación e internet en la práctica profesional pone de relieve la importancia de la interacción de las variables relacionadas con el contexto organizativo de uso de estas tecnologías (17).

En el caso de la telemedicina, además de las variables relacionadas con el contexto organizativo, también observamos la importancia de las variables relacionadas con las características formativas de los individuos y su experiencia. Si internet era utilizado con mayor medida por los profesionales más jóvenes, en el acceso a las tecnologías relacionadas con la telemedicina destacan los profesionales más maduros que desarrollan su labor en hospitales. Estos profesionales tienen acceso a este tipo de tecnologías dada su experiencia, los méritos logrados y la posición jerárquica que pueden tener dentro de su institución.

En general es en los hospitales donde se utilizan estas tecnologías, ya que son el tipo de instituciones que pueden tener la capacidad de inversión necesaria. Además, en muchas ocasiones las estructuras jerárquicas de estos centros dotan de un gran poder a los profesionales de más prestigio, lo que facilita su autonomía a la hora de decidir qué tipo de tecnologías se utilizan en sus servicios.

El uso de las tecnologías de la información y la comunicación para la práctica de la telemedicina afecta positivamente al prestigio de los profesionales, por tanto, este uso está asociado con una visión positiva de la tecnología.

En la valoración de los usos de internet por parte de los profesionales destaca la idea de que la introducción y uso de este tipo de tecnologías no pueden abordarse desde una perspectiva determinista. Es decir, los profesionales son conscientes de que en estos procesos no se produce una adaptación de sus conductas automáticamente, sino que se producen interacciones entre factores humanos, culturales, organizativos y tecnológicos que moldean tanto su conducta como la propia tecnología.

En general, los profesionales médicos de unidades de dolor tienen una percepción positiva del uso de las tecnologías de la información y la comunicación e internet en su actividad profesional.

Esta visión queda matizada fundamentalmente por dos cuestiones.

En primer lugar, por el uso que los pacientes pueden hacer de estas tecnologías y por las consecuencias que de este uso puedan derivarse sobre la relación médicopaciente y sobre la salud del propio paciente.

En segundo lugar, esta visión positiva se ha de enfrentar a las complejas interacciones que suponen la introducción y uso de estas tecnologías en los centros donde desarrollan su actividad, ya que esto viene acompañado de una reestructuración social y organizativa que afecta directamente a sus actividades cotidianas.

En el caso de los profesionales encuestados, las variables que más peso tienen sobre este impacto no son las relacionadas con las características de los individuos (formación, habilidades de aprendizaje y experiencia] sino aquellas variables que tienen relación con sus lugares de trabajo, con la relación de los centros proveedores de servicios con su entorno y con el patrón de competitividad económico y social de estos.

Esto se pone de manifiesto tanto en la identificación y caracterización del médico en unidades de dolor como en sus determinantes.

Por último, la aplicación de las tecnologías de la información y la comunicación a la medicina y a la asistencia sanitaria (telemedicina-teleasistencia) ha permitido la prestación remota de servicios médicos y asistenciales. Este hecho ha provocado que desde las esferas políticas se le preste gran atención a las posibilidades de estas tecnologías en relación con los problemas de acceso a los servicios sanitarios y como herramienta que facilite la gestión de la demanda, especialmente en el acceso a servicios sanitarios especializados, como es el caso de unidades de dolor (18).

Se observa que existe un porcentaje mayor de lo esperado de médico en unidades de dolor entre los hombres, entre los mayores de 40 años, entre los que trabajan en un hospital, entre los que trabajan en más de un centro asistencial cuya titularidad es mixta (pública/privada), entre aquellos que realizan más actividades que la práctica asistencial, entre aquellos que utilizan con más intensidad internet, entre aquellos que tienen más años de experiencia en el uso de internet y encuentran esta tecnología más útil, entre aquellos que valoran más positivamente la información disponible en internet y dialogan con los pacientes sobre esta fuente de información. Además, también existe un porcentaje mayor de lo esperado de médicos en unidad de dolor entre aquellos que valoran más positivamente las consecuencias de esta tecnología sobre la mejora de la relación y la comunicación con sus pacientes, la eficiencia y productividad de su trabajo. Finalmente, existe un porcentaje mayor de lo esperado de este nuevo perfil profesional entre aquellos médicos que hacen un uso intensivo de los sistemas de información y valoran más positivamente el impacto de las tecnologías de la información y la comunicación en su práctica clínica. 


\section{AGRADECIMIENTOS}

A los alumnos del Máster del Dolor de la USAL por suministrarnos los datos para el estudio.

\section{CONFLICTO DE INTERESES}

En ningún caso la financiación del proyecto ha supuesto un condicionante o limitación a la total independencia de la investigación.

\section{BIBLIOGRAFÍA}

1. OECD. Information Technology Outlook. [artículo en línea]. OCDE. [Consultado el 25 de septiembre de 2018]. http:// www. oecd.org/dataoecd/20/47/33951035.pdf.

2. Anderson GF, Frogner BK, Johns RA, Reinhardt UE. Health care spending and use of information technology in OECD countries. Health Affairs 2006;25(3):819-31. DOI: 10.1377/ hlthaff.25.3.819.

3. Burrows R, Nettleton S, Pleace N. Virtual community care? Social policy and the mergence of computer mediated social support. Information, Communication \& Society 2000;3(1):95-121. DOl: 10.1080/136911800359446.

4. Kassirer JP. Patients, physicians, and the internet. Health Affairs 2000;19(6):115- 23.

5. Miller TE, Derse AR. Between strangers: the practice of medicine online. Health Affairs 2002;21(4):168-79.

6. Levy J, Strombeck R. Health benefits and risk of the internet. J Med Syst 2002;26(6):495-510.

7. Lorenzo S, Mira JJ. Are Spanish physicians ready to take advantage of the Internet? World Hospitals and Health Services 2004;40(3):31-5.

8. Hasty RT, Garbalosa RC, Barbato VA, Valdes PJ Jr, Powers DW, Hernandez E, et al. Wikipedia vs. peer-reviewed medical literature foro information about the 10 most costly medical conditions. J Am Osteopath Assoc 2014;114(5):368-73. DOI: 10.7556/jaoa.2014.035.
9. Love T, Laier C, Brand M, Hatch L, Hajela R. Neuroscience of Internet Addiction: A Review and Update. Behav Sci 2015;5(3):388-433. DOI: 10.3390/bs5030388.

10. Heath C, Luff P, Sanchez M. Technology and medical practice. Sociol Health IIIn 2003;25:75-96.

11. Muriel J, Sánchez Ledesma MJ, López Millan JM, García-Cenador MB. Study of the uses of Information and Communication Technologies by Pain Treatment Unit Physicians. J Med Syst 2017;41(5):78-86. DOl: 10.1007/s10916-017-0726-3.

12. Chew F, Grant W, Totes R. Doctors on-line: using diffusion of innovations theory to understand internet use. Fam Med 2004;36(9):645-50.

13. Dear BF, Gandy M, Karin E, Staples LG, Johnston L, Fogliati VJ, et al. The Pain Course: a randomised controlled trial examining an internet-delivered pain management program when provided with different levels of clinician support. Pain 2015;156(10):1920-35. DOI: 10.1097/j. pain.0000000000000251.

14. Muriel J, García-Cenador MB, López Millan JM, Juanes JA, Sánchez-Ledesma MJ. Use of Information and Communication Technologies in Clinical Practice Related to the Treatment of Pain. Influence on the Professional Activity and the Doctor-Patient Relationship. J Med Syst 2017;41(5):7782. DOI: 10.1007/s10916-017-0724-5.

15. Cullen RJ. In search of evidence: family practicionaers' use of Internet for clinical information. J Med Libr Assoc 2002;90(4):370-9.

16. Wilkinson JF, Short HL, Wilkinson S, Mander A. Commissioning for menopause specialist services: A local perspective: An internet-based survey to assess the potential demand for menopause care in West Cheshire and the skills of local primary care clinicians in this field, with a view to informing future commissioning locally. Post Reprod Health 2015;21(3):98104. DOI: 10.1177/2053369115595309.

17. Walsh K. Neuroscience education on the internet: the next steps. Malays J Med Sci 2014;21(6);75-82.

18. De Lima Verde Brito TD, Baptista RS, de Lima Lopes PR, Haddad AE, Messina LA, Torres Pisa I. The Collaborative Coordination of Special Interest Groups on the Telemedicine University Network (RUTE) in Brazil.Stud Health Technol Inform. 2015;216:1010. 